\title{
Testing social robot acceptance: what if you could be assessed for dementia by a robot? A pilot study.
}

\author{
Lucie Cormons \\ Univ. Grenoble Alpes, LIP/PC2S \\ 38000 Grenoble \\ France \\ +33(0)4768278 12
}

lucie.cormons@univ-grenoble-alpes.fr

\author{
Damien Pellier \\ Univ. Grenoble Alpes, LIG \\ 38000 Grenoble \\ France \\ +33(0)457421543
}

damien.pellier@univ-grenoble-alpes.fr

\author{
Caroline Poulet \\ Univ. Grenoble Alpes, LIP/PC2S \\ 38000 Grenoble \\ France \\ +33(0)4 76827812 \\ Caroline.poulet@univ-grenoble-alpes.fr
}

\author{
Humbert Fiorino \\ Univ. Grenoble Alpes, LIG \\ 38000 Grenoble \\ France \\ +33(0)457421543
}

humbert.fiorino@univ-grenoble-alpes.fr

\author{
Sylvie Pesty \\ Univ. Grenoble Alpes, LIG \\ 38000 Grenoble \\ France \\ +33 (0)457421543 \\ sylvie.pesty@univ-grenoble-alpes.fr
}

\begin{abstract}
It is beneficial to identify and begin treatment of neurocognitive disorders of the elderly as early as possible. In order to help diagnose these disorders, social assistive robots are promising technologies to assist psychologists. To be accepted by the elderly, the robot behaviours must be close enough to the fundamental competences of the psychologists in order not to confuse the patient. This pilot study aims (1) to design a social assistive robot capable of performing a memory evaluation test, (2) to gather opinions on the robot's acceptability with an innovative method (persona) and (3) to identify robot behavioural improvements. We used the "persona methodology" for this pilot study. A panel of students playing the role of a "persona" performed the memory test called RL/RI16, by interacting with the social robot Pepper and then were interviewed about their experience. The robot plays the psychologist role. The interviews and videos analysis showed that the robot is not yet well accepted but the analysis results gave interesting leads to continue.
\end{abstract}

Keywords: social Human-Robot Interaction (HRI), assistive robotics; acceptability of robots by the elderly; "persona methodology".

\section{INTRODUCTION}

According to data from World Population Prospects ${ }^{1}$, by 2050 , one in six people in the world will be over age $65(16 \%)$, up from one in eleven in $2019(9 \%)$. The number of persons aged 80 years or over is projected to triple, from 143 million in 2019 to 426 million in 2050. The more the elderly population increases the more that population is likely to suffer from Major Neurocognitive Disorders (MNDs) like Alzheimer. MNDs affect 47 million people worldwide [1]. In order to improve patient management, cognitive deficits must be detected early using neuropsychological tests performed by psychologists or neuropsychologists [2].

Unfortunately, the elderly are often not diagnosed in time [3]. Society must reconsider the health and social care system to meet the new challenges of an ageing population, especially in terms of major neurocognitive disorder (such as Alzheimer) screening. To fight this issue, a virtual revolution has taken place for clinical purpose including the implementation of social robots within the health care context [4]. Combining also with the shortage of physicians in some parts of the territory, robots may provide important aid to overburdened health-care providers [5]. Robots appear to be accepted by patients, especially if they demonstrate social skills [6][7][8], but the qualities required for robots to be highly accepted by patients are not yet well understood. Furthermore, several studies have examined both companion type robots and service type robots interacting with elderly but few have analysed the acceptability of robots during a more professional task. A lack of studies regarding robots performing more health-oriented tasks is to be noted in the current literature. This knowledge gap motives the current research on acceptability of robots playing the role of a health-care professional.

As the elderly will interact with the robot for a memory problem screening test, which is stressful in itself, particular care must be taken to provide the robot with appropriate socio-communicative skills. The ingredients to promote the acceptance of robots by the elderly are a current hot topic [9]. For now, recent studies show the importance of robot social behaviour even more so in a health- and eldercare environment. In other words, robots that possess better social skills would be able to communicate more effectively, being judged as more pleasant and therefore would be more acceptable during interactions [6]. Also, programming a robot to interact with an elderly person requires multiple important factors. Indeed, elderly can require more effort than 
younger patients in terms of communication due to aging. The elderly are more likely to suffer from hearing loss, vision loss [10], attention loss [11] and have more difficulty speaking, especially in louder tones [12]. Therefore, considering the health care situation, the special needs of the elderly might inform the design of robots intended for such an audience.

The pilot study presented in this paper has three main objectives. First, it aims to create a socially assistive robot capable of performing a memory test based on a real psychologist / elderly person interaction script. Second, it aims to analyse the acceptability of the robot with an innovative methodology (persona). Third, it aims to get participant feedback on robot behavioural improvements, as well as to gain insight regarding the task performance and social cues necessary to increase acceptability.

\section{RELATED WORK}

\section{A. Robots as a tool for eldercare}

A growing number of projects address the development of robots for experiments in eldercare. Most of the time, two kinds of experiments are conducted with the elderly suffering from neurocognitive impairment: some use companion and/or mediation robots like Paro (seal shaped robot) [13] where the focus is on social acceptance, while others use service type social robots like iCat [6] [8]. The latter studies focused more on acceptance of the robot regarding its basic functionalities, like asking the participant whether they were adhering to their activity plan [7]. Most of the studies on robotic acceptance showed that the elderly are more comfortable with a more sociable robot and behaviour analysis shows that the elderly are invited to be more expressive by a more sociable robot [14][15][7].

Some other experiments were conducted where the robot takes the place not of a companion, but of a professional. [16] did a study for the elderly suffering from body impairment where the robot was designed to help them in their rehabilitation journey. Participants indicate that they perceived the robot to be moderately intelligent, and to be moderately helpful with the exercises. The robot was seen by the participants to be an entity that they could trust and that could help them, rather than just entertaining them. More recently, a team conducted a study where patients were diagnosed with depression by either a psychiatrist or an Embodied Conversational Agents (ECA) [5]. Patients found the face-to-face interview with the ECA very acceptable, suggesting that ECA "can communicate empathy, elicit patient trust, reduce the feeling of being judged by a human and reduce emotional barriers to disclose an affective state" (p.5). With the same idea, [17] proposed the SimCoach project involving a virtual agent to identify symptoms of depression, anxiety, and PostTraumatic Stress Disorder (PTSD). The authors aim to break down barriers to care like stigma or unawareness by providing confidential help, and to promote the initiation to psychological healthcare information and assistance to both military personnel and family members.

\section{B. Measuring robot acceptability for the elderly}

Several models exist to measure the acceptability of a new technology or, more specifically, to measure the intention to adopt a new technology [18]. Primarily based on the theory of planned behaviour of [19], the first model to measure acceptance, named the Technology Acceptance Model (TAM), was developed [20]. Afterwards, [18] offered an overview of these later technology acceptance models and incorporated the most reliable constructs into the Unified Theory of Acceptance and Use of Technology (UTAUT). The UTAUT aims to explain user intentions to use an information system and subsequent usage behaviour. The theory consists of four key constructs, which are performance expectancy, effort expectancy, social influence, and facilitating conditions. The acceptability is moderated by gender, age, experience and voluntariness of use. However, traditional technology acceptance models do not consider a crucial aspect: the social abilities of the robot and its impact on the interaction. Interaction is, unsurprisingly, a very important aspect of the process of acceptability when it comes to social robotics. In order to address these issues, the Almere model was created [7]. This model is the adaptation and the theoretical extension of the UTAUT by explaining intent to use not only in terms of variables related to functional evaluation like perceived usefulness and perceived ease of use, but also variables that relate to social interaction. Heerink added variables like perceived enjoyment, defined as the feeling of joy or pleasure associated by the user with the use of the robot, and social presence, defined as the experience of sensing a social entity when interacting with the robot. [6] said that "a user accepts the robot as a conversational partner, finds the robot's social skills credible, sees the robot as an autonomous social being and is more likely to exhibit natural verbal and nonverbal conversational behaviour as well as feeling comfortable in interacting with the robot". In return, the user will demonstrate more conversational engagement by being more expressive [21] and thus we can use behavioural clues as an indication of conversational acceptance [22]. At the end, The Almere model counts 13 variables: anxiety, attitude towards technology, facilitating conditions, intention to use, perceived adaptiveness, perceived enjoyment, perceived ease of use, perceived sociability, perceived usefulness, social influence, social presence, trust, use.

Presently, research on robot acceptability can be divided into two main areas: functional acceptance (usefulness and ease of use) and social acceptance (acceptance of the robot as a conversational partner) [14]. 
It seems important to open the spectrum of research to include these constructs in the context of robots with professional health care tasks.

\section{Methodology}

\section{A. Overall methodology}

The robot we used is Pepper, a semi-humanoid robot manufactured by Softbank Robotics. Pepper is 1.20 meters tall and has a screen on his chest that allows transmitting written content.

\section{1) Dialog scripts creation for the Pepper robot}

First, we created an audio-visual corpus where a psychologist $(\mathrm{N}=3)$ interacts with an elderly person $(\mathrm{N}=14)$ for performing the RL/RI16 memory test. The RL/RI16 memory test (for Rappel Libre/Rappel indicé in French) is an adaptation of an English test (Free and Cued Selective Reminding - FCSR test) [23]. It is used widely to identify memory loss. It is structured in five major and well-defined steps, which is an undeniable advantage for robot programming. The test is composed of 16 words to be memorized. The words belong to 16 different semantic categories. Usually, a psychologist performs the test using a pencil, a stopwatch and four cardboards on which are written the 16 words to be learned four by four. The test duration is about 20 minutes.

From the corpus transcription (the 14 PsychologistElder dialogs), we analysed the psychologists' sociocommunicative (SC) used to perform the RL/RI16. Two major trends of SC competencies were identified: SC that mainly focus on the task and SC that show mainly focus on the relationship with the patient. We thus have divided the psychologists' SC competencies in two main groups: task-oriented (e.g.: to inform, to give the correction, to explain, etc.) or relational-oriented (e.g.: to be sympathetic, to be reassuring, to motivate the patient).

For the pilot study we present in this paper, we used the most task-oriented dialog to create a prototypical script-dialog to be implemented. We decided to choose for the robot the task-oriented script in order to have a strong base to be implemented that could be compared later with a relation-oriented script more difficult to implement because it requires more behavioural repertoires, laughs, different tones that are more complex to implement on the present version of Pepper. Moreover, as the task-oriented script consists in a tighter basis, it will allow us to identify what behaviours are missing and what are mandatory. By time economy for the participants, we only perform the three main steps of the RL/RI16 test and limited the test to eight words to be memorized (mean time $=5 \mathrm{~min} 45 ;$ S.D. $=0.28 \mathrm{sec}$ ).

\section{2) Innovative methodology (persona)}

It is always difficult to recruit people for robotic experiments, especially fragile participants (e.g.: the elderly). However, even if the right participants are difficult to recruit, there is still a crucial need to coconstruct with those users.

We therefore choose to use "the persona methodology" for the robotic experimentation that is a reasonable compromise because personas represent possible users that designers can refer to when the targeted population is not available. This methodology consists in creating fictive persons with attributes, social and psychological characteristic to represent a targeted population. A persona therefore represents a user with a name, a face, a personal history and values, which gives him a certain humanity [24]. Personas should not be stereotypical, but complex enough and realistic to be effective [24]. These "cognitive instruments" helps to think and guide decisions about the design of multiple products and services. Even though the "persona methodology" does not physically connect designers and users, it makes available representations of future users.

By following the "persona methodology", two personas were created. The first one represented Pierre, a 75 years old man who lives in a retired house. Pierre is lonely and has a lack of autonomy; he suffered from minor neurocognitive issues, especially memory loss and communication difficulties (lack of concentration and lack of fluency). The second persona was Mireille, an active and dynamic 69 years old elderly woman, with no specific issue. A document with the person picture, name, story, characteristics were handed to the participants.

\section{B. Procedure}

\section{1) Pre-test}

A pre-test has been performed to verify if both the two scripts-dialogs were correctly identified as either taskoriented or relation-oriented. Two actors were recorded for the task-oriented script, one actor was playing the role of the psychologist, the other one was playing the role of a patient. Then we recorded the same two persons acting the relationoriented script. An online questionnaire was created with video segments. Nineteen students of our university replied on a Lickert-scale (1 to 5 points) with two extremities from "relational-oriented" to "task-oriented". After making sure that the task-oriented was correctly identified by participants, the script was programmed in Pepper.

\section{2) Test}

For two days, seven students from our university came to the laboratory. The experimenter presented the persona to the participant and asked him/her to feel like he/she was the persona, to understand the persona specificity and needs. The consign were strictly assigned to the participant, and the experimentation precautionary make sure that the participant understood his role. After the signature of legal documents (informed consent form, image rights form), the participant was invited to seat in front of Pepper. Two engineers were asked to stay in the room in case of issues to give some 
instruction to the participant (speak loud enough, clearly, repeat if it's necessary, speak when the eyes of Pepper are light up, etc.). The cameras were tuned on and the dialog Human-Pepper started. Pepper ran the RL/RI16 test ${ }^{2}$ asking questions to the participant and waiting for answers (see figure 1). After the interaction with Pepper the participants replied to a semi-structured and audio-recorded interviews (mean time $=21,43 \mathrm{~min}$ ). The interview focused on questions to gather the perceived pros and cons to the robot usage, on both the robot abilities to interact and to its abilities to perform the test, on what changes could be applied to make the robot more efficient to interact with the elderly, and on what emotions the interactions triggered. Finally, some additional socio-demographic questions were asked. The questions find a theoretical support on the Almere model [7]. The participants had to reply accordingly to their persona conditions (Pierre, Mireille, no persona).

An intentional choice was made regarding the few numbers of participants. Qualitative data were privileged to gather an important set of feedbacks and strong ergonomic recommendations for further robot programming. These types of analysis do not require a huge sample because it privileges the quality over the quantity. Also, data analysis (transcription, content analysis and video analysis) is a timeconsuming process.

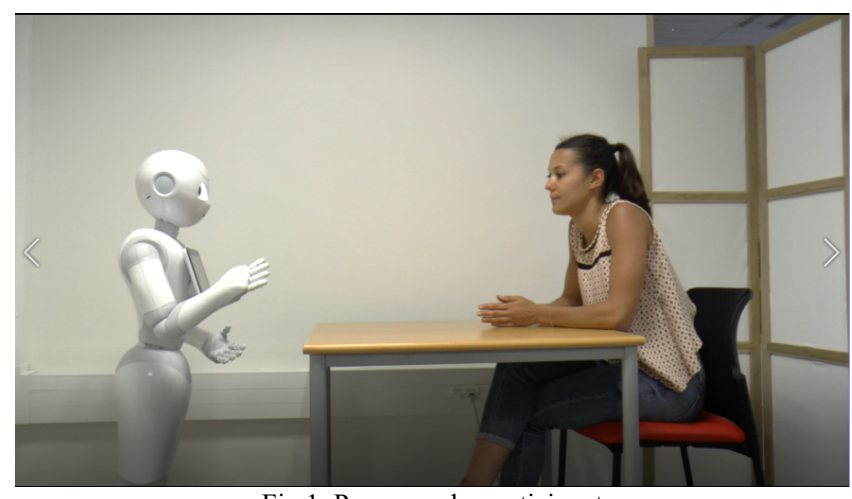

Fig.1. Pepper and a participant

\section{3) Data analysis}

Both the interviews and the video of the interaction were analysed. The interviews were transcribed, and a content analysis was performed. To performed the content analysis, we used the 13 variables of the Almere Model as a grid analysis to see if the verbatim would fit with the model [7]. The videos were analysed via an analysis grid inspired from the list of social behaviours of [15] like nodding head, shaking head; verbal and non-verbal greeting; moving away from the robot; moving forwards the robot; smiling; laughing; Showing irritation; looking surprised. We decided to add two categories that quantify Pepper difficulties to interact and the necessity for the participant to speak loudly to be correctly understand by Pepper.

\section{RESULTS AND DISCUSSION}

\section{1) Pre-test}

Nineteen participants from 18 and 38 years old were asked to judge video segment (task-oriented $v s$ relationaloriented) of actors doing the RL/RI16 test. Participants correctly answered and recognised that the segment videos were task-oriented or relational-oriented $(63.2 \%$ of correct answer for the relational-oriented video segment; $97.4 \%$ of correct answer for the task-oriented video segment).

\section{2) Test}

Seven non computer scientist students $(N=4$ women, mean age $=21,4, S . D .=0.92)$ interacted with Pepper. Two students had to play the role of the persona $\mathrm{n}^{\circ} 1$ (Mireille), two had the persona ${ }^{\circ} 2$ (Pierre Legrand), and three had no persona at all.

\section{Video analysis:}

Most of the participants greeted sympathetically Pepper at the beginning and at the end of the session (4 out of 7). While Pepper was giving the instructions, 5 participants expressed their understanding by nodding (mean of 2 times each). Regarding the interpersonal distance between Pepper and the participant, the video analysis showed that 4 participants reduced the distance between them and the Pepper by moving forwards it, 3 participants had a neutral position (not forward either backward). These results show quite a lot of social behaviours especially by nodding. No one moved away from Pepper.

The robot having some bugs due to its sensors (mainly it doesn't correctly ear the participants, consequently it repeats itself many times) we analysed the persons' reactions. They mostly react by smiling or laughing. In cases of repeated bugs in short times, participants show some irritation or surprise by frowning or raising their eyebrows.

Surprisingly, even though the task was easy (mainly 8 words to be remembered from pictures) and the participants were young, highly educated students (mostly $4^{\text {th }}$ years of study), they did not succeed in completing the memory task correctly. The mean score of words correctly recalled were 5.4/8. In the interviews, participants never declare that they did poorly at the task, but they said that interacting with the robot ask them a certain cognitive effort. The fact they had to focus more on interacting with the robot (waiting for Pepper's eyes light up before answering, speaking out clearly, etc.) requires cognitive effort and it potentially explains why they did poorly at the memory task. These findings suggest that the memory test is yet a tricky task to be performed by a robot as the Pepper robot. This has impacted the robot interactional performance and costs cognitive effort for the participants and reduce their own test performance. It's clear that a robot performing a memory test must be of high quality, hearing correctly, answering without error and the dialogue must be fluid.

\footnotetext{
${ }^{2}$ An interaction sample:

https://www.youtube.com/watch?v=OHSQXxAEkJc
} 


\section{Content analysis:}

When we ask the participants' opinion on having a test being done by a robot, we can observe both strengths and weaknesses. On the one hand, Pepper suffers from critics especially because of its technical defects that have negative impact on test performance. Participants reported maintaining a high level of concentration when talking to Pepper because they must focus both on Pepper's eyes colour to know when to talk (4 times) and on speaking loudly because of Pepper poor hearing capacities ( 8 times). Theses cognitive efforts are likely to impact participants test performance (especially on a memory task where focus is particularly needed). To this point, one participant declares "I was focused on how the robot was reacting to know when to talk, so I would forget the words". Furthermore, the participants judge that the robot is missing three different human aspects that limit the robot usage. First, the lack of communication behaviours (10 times) (e.g.: "I need to put on more effort to understand Pepper"), second, the lack of adaptability and behavioural repertoire (5 times) (e.g. "Pepper always say the same things") and finally, the lack of emotional behaviours (4 times) ("Pepper isn't warm enough"). In summary, participants complain mainly that the robot can't "understand" the person, can't personalize his speech, and doesn't show any reactions nor emotions. Also, participants criticized six times the robot's lack of relational and pedagogic attributes. It's partly due to the task-oriented script we implemented which definitively lacks of relational abilities. Five times participants will point out the restricted behavioural repertoire even if we gave Pepper a repertoire of various responses. Participants expressed that the elderly are likely to ask questions or have atypical behaviours which will challenge the robot. The last negative point is the robot's poor possibility to express emotions. The participants clearly express the need of capturing the interlocutor's emotions to feel comfortable within an interaction (e.g., "with a human being I understand, at least I try to understand, what he thinks through his gaze, his facial expressions, the tone used, but with a robot it is always the same"). Participants explain that the robot seems not understanding participants' social signal in order to adapt its behaviour. They wanted the robot understands if they need help, motivation, or if they are thinking and they need time to focus, etc. Participants wanted to know if they are right or wrong by looking at the robot's expressions. In other words, it seems that they want feedback on their own performance during the interaction. For example, participants repeatedly expressed that the robot should cue their correctness during the task quickly.

In contrast, participants exposed some advantages like they would feel comfortable because they won't feel judge by the robot Pepper so they would feel more comfortable making mistakes (4 times) and they would have no time pressure to reply to the exercise (5 times). One participant underlies that having a robot doing the test may improve test standardization. Then, participants perceived the sociability aspects of Pepper that helped test performance, like humans' interactional attributes that make him friendly (e.g., head and body movements) (7 times). Pepper is also judged as possessing both technical ("it is clear") (3 times) and social psychologist skills ("he looks kind which put you in good conditions to complete the task" or "it makes you want to do the test.") (2 times). The ergonomic aspects were very much appreciated, Pepper was complimented 7 times as being attractive with a great, nice, and functional screen. Seven times participants alluded to what Heerink, Kröse, Evers, and Wielinga (2010) called the perceived sociability that is the "the perceived ability of the system to perform sociable behaviour" (p.4). The perceived sociability positively impacts the interaction quality because participants felt no time pressure to reply to the robot and that the robot had a friendly tone.

By looking deeper into the results, we observed that participants are simultaneously impacted by the technical defects and appreciative of the sociable behaviours of the robot. They referred to the defects that impair the relationship 7 times. For example, participants had to speak loudly and repeat their statements several times before the robot understood. A concern was made on the fact that the robot can't reply to questions which can be an important source of confusion in the case a patient does not understand the instruction. This concern is increased for people suffering from mid-cognitive impairment because due to their condition, they would need more help and more explanation than someone with no disorder (Pierre-condition group).

Interacting with a robot isn't a common thing. It can be a source of negative emotions for some participants (more precisely, uncomfortable ( 3 out of 7 participants), stressed ( 3 out of 7), and fearful ( 1 out of 7) even though 3 participants described having perceived it to be friendly, funny and pleasant. Finally, all the participants questioned the ability of a robot to perform a memory test to an elderly person. Three times, participants suggest that the robot could be an accessory to a psychologist by helping him not to make mistakes.

Finally, if we analyse the results per group, the Mireillecondition and no persona condition reveal quite the same results in the overall content analysis. The Pierre-condition group referred to the sociable behaviours (7 times). For example, it seemed sympathetic (especially his tone), the participants felt they had the time to reply, and the instructions were clear. The Pierre-condition group were particularly satisfied with the screen which augmented the audio information provided by Pepper's speech with a visual aid (an image illustrates the word to be memorized, like in the real test). They expressed that they read the words properly and without difficulties. Furthermore, they were amused by the robot character as new and intriguing.

\section{CONCLUSION AND PERSPECTIVES}

In this pilot study we have presented the design and evaluation of a socially assistive robot that can interact with an elderly person and engage the person in a memory test. 
We defined a task-oriented script dialog implemented in the robot based on real psychologists' dialogs in situation of performing the RL/RI16 test. A feasibility study was conducted with the innovative "persona methodology". The results do not validate the acceptability of the assistive robot in being able to perform a memory test to the elderly. Nevertheless, the pilot study gave us a lot of points to work on. The content analysis reflects that even if participants felt it was fun or pleasant to be assessed by a robot, the robot suffer from technical defects decreasing its acceptability. The major positive factor was that participants did not feel judged by the robot and felt they could take more time to reply or make mistakes. The control group and the Mireillegroup condition gave quite similar answers. This result leads to think that an effort in terms of programming a warm, attentive robot with a large set of behavioural repertoires should be made especially when the patients are suffering cognitive impairment. The main fear for the three groups was that the robot would not be able to reply to the questions or understand that the patient has trouble understanding the test instructions like it might be the case if the elderly is suffering from mid-cognitive impairment. Participants recognize qualities to Pepper, but we can observe that the positive sentences are often punctuated with a "but" that is generally introducing a negative opinion (e.g.: it will never replace a human). Pepper needs ergonomic improvements specially to interact with an elderly person who might suffer from sensorial and cognitive impairment. Regarding improvement, participants uttered the need for turn-taking management, and for speech adaptation to the user, especially if the user possesses particularity (low cognitive level). The communication behaviour repertoire should be extended even for standardized tests (e.g.: RL/RI16). The robot hearing capacity needs to be improved. Finally, relational skills and positive non-verbal expressions in reaction to what the person is saying, need to be added. This last wish seems to show that people need feedback from the robot on their own behaviour to adapt themselves to the robot ("with a human, if I am wrong, I would have seen it on the psychologist face. With the robot, I had no information on how I was doing"). The video analysis showed that people were optimistic and patient with the robot even though the robot was not interacting in an optimal way. We are convinced that the relation-oriented script dialog, which includes positive feedbacks to the elderly, nod of the head, encouragements, etc. will already be much better.

This pilot study also wanted to challenge a new way of testing acceptability with the "persona methodology". [25] underlies that the field is young and that method for measuring acceptability of social assistive robots as to be developed and open to new methodology. This "persona methodology" allowed us to conduct our study even though the robot was imperfect and elderly persons not available, and gave us important points to work on.

In conclusion, new technologies will be required in years to support psychologists who screen for Major
Neurocognitive Disorders (MNDs). Therefore, research and especially the collaborative work between informatic science and psychology should continue to address the issues raised in this pilot study. Such robotic solutions could be very useful in helping psychologists to diagnose MNDs diseases as early as possible. The growing interested in electronic-health (E-health) solutions brings up a main issue coming along with it: the robotic acceptance. In our case, we need to focus on how to provide a quality interaction between elderly that potentially suffer from mid-cognitive impairment and a robot. Starting by the analysis of real interaction allows the programmers to better understand the interactional needs required to perform the task efficiently and promote acceptability. But being assessed for dementia by a robot also raise considerable ethic consideration and this issue will undoubtedly need to be addressed.

\section{ACKNOWLEDGMENT}

This research was supported by the French funded ANR project: "Embodying socio-communicative behaviours in humanoid robots" (ANR-14-CE27-0014). This research has been also supervised by Michel Dubois, Professor in Work and Organizational Psychology at LIP/PC2S, UGA who passed away in July 2018. This work could not have been done without his extreme kindness and expertise.

\section{REFERENCES}

[1] World Human Organization (2016). Rapport mondial sur le vieillissement et la santé.

[2] Pasquier, F. (1999). Early diagnosis of dementia: Neuropsychology. Journal of neurology, 246(1), 6-15.

[3] Iliffe, S., Manthorpe, J., \& Eden, A. (2003). Sooner or later? Issues in the early diagnosis of dementia in general practice: a qualitative study. Family Practice, 20(4), 376-381.

[4] Walker, R. E., \& Foley, J. M. (1973). Social intelligence: Its history and measurement. Psychological Reports, 33(3), 839864.

[5] Philip, P., Micoulaud-Franchi, J.-A., Sagaspe, P., De Sevin, E., Olive, J., Bioulac, S., \& Sauteraud, A. (2017). Virtual human as a new diagnostic tool, a proof of concept study in the field of major depressive disorders. Scientific reports, 7, 42656.

[6] Heerink, M., Kröse, B., Wielinga, B. J., \& Evers, V. (2006). Studying the acceptance of a robotic agent by elderly users. International Journal of Assistive Robotics and Mechatronics, 7(3), 33-43.

[7] Heerink, M., Kröse, B., Evers, V., \& Wielinga, B. (2010). Assessing acceptance of assistive social agent technology by older adults: The almere model. International journal of social robotics, 2(4), 361-375.

[8] Klamer, T., \& Allouch, S. B. (2010). Acceptance and use of a social robot by elderly users in a domestic environment. 2010 4th International Conference on Pervasive Computing Technologies for Healthcare, 1-8. IEEE.

[9] Caulfield, B. M., \& Donnelly, S. C. (2013). What is Connected Health and why will it change your practice? QJM: An International Journal of Medicine, 106(8), 703-707. 
[10] Covelet, R. (2007). Prendre enfin conscience des enjeux des déficits sensoriels des personnes âgées. Gérontologie et société, 30(4), 249-262

[11] Salthouse, T. A. (2010). Selective review of cognitive aging. Journal of the International neuropsychological Society, 16(5), 754-760.

[12] Salthouse, T. A. (2004). What and when of cognitive aging. Current directions in psychological science, 13(4), 140-144.

[13] Shibata, T., \& Wada, K. (2011). Robot therapy: A new approach for mental healthcare of the elderly-a mini-review. Gerontology, 57(4), 378-386.

[14] Heerink, Marcel, Kröse, B., Wielinga, B., \& Evers, V. (2008). Enjoyment intention to use and actual use of a conversational robot by elderly people. Proceedings of the 3rd ACM/IEEE international conference on Human robot interaction, 113120. ACM.

[15] De Ruyter, B., Saini, P., Markopoulos, P., \& Van Breemen, A. (2005). Assessing the effects of building social intelligence in a robotic interface for the home. Interacting with computers, 17(5), 522-541.

[16] Fasola, J., \& Mataric, M. J. (2010). Robot exercise instructor: A socially assistive robot system to monitor and encourage physical exercise for the elderly. 19th International Symposium in Robot and Human Interactive Communication, 416-421. IEEE.

[17] Rizzo, A., Lange, B., Buckwalter, J. G., Forbell, E., Kim, J., Sagae, K., et al. (2011). SimCoach: An intelligent virtual human system for providing healthcare information and support. International Journal on Disability and Human Development, 10(4), 277-281.

[18] Venkatesh, V., Morris, M. G., Davis, G. B., \& Davis, F. D. (2003). User acceptance of information technology: Toward a unified view. MIS quarterly, 425-478.

[19] Ajzen, I. (1991). The theory of planned behavior. Organizational behavior and human decision processes, 50(2), 179-211.

[20] Davis, F. D. (1989). Perceived usefulness, perceived ease of use, and user acceptance of information technology. MIS quarterly, 319-340.

[21] Axelrod, L., \& Hone, K. (2005). Identifying Affectemes: Transcribing Conversational Behaviour. Informatics for Supporting Social Intelligence and, 64 .

[22] Sidner, C. L., Lee, C., \& Kidd, C. (2005). Engagement during dialogues with robots. AAAI spring symposia.

[23] Van der Linden, M., Coyette, F., Poitrenaud, J., Kalafat, M., Calicis, F., Wyns, C., \& Adam, S. (2004). L'épreuve de rappel libre/rappel indicé à 16 items (RL/RI-16)

[24] Bornet, C., \& Brangier, E. (2013). La méthode des personas : Principes, intérêts et limites. Bulletin de psychologie, (2), 115134.

[25] Tapus, A., Maja, M., \& Scassellatti, B. (2007). The grand challenges in socially assistive robotics. 